\title{
Familial progressive hyper- and hypopigmentation: a report on a Chinese family and evidence for genetic heterogeneity ${ }^{*}$
}

\author{
Fang Xiao-Kai ${ }^{1}$ \\ Li Yan-Jia ${ }^{2}$ \\ Wang He-Peng ${ }^{2}$
}

\author{
He Yue-Xi ${ }^{1}$ \\ Chen Li-Rong ${ }^{2}$ \\ Sun Qing ${ }^{3}$
}

DOI: http:/ /dx.doi.org/10.1590/abd1806-4841.20175567

\begin{abstract}
BACKGROUND: Familial progressive hyper- and hypopigmentation (FPHH) is a rare genodermatosis that is characterized by diffuse hyper- and hypopigmented spots on the skin and mucous membranes. It is caused by a pathogenic mutation of the KITLG gene.

Овјестіves: To investigate the clinical features and mutation of the KITLG gene in a Chinese family with FPHH.

METHODS: Histopathological and immunohistochemical analysis of lesions from the proband was performed. The KITLG gene was screened for the presence of mutations.

RESUlTs: A Chinese family containing 14 individuals with FPHH was described, and the proband was a 5-year-old girl showing diffuse hyper- and hypopigmented lesions on her extremities and trunk. Histopathological and immunohistochemical staining for S100 and HMB45 of skin biopsy specimens from the hyperpigmented areas showed a striking increase in melanin throughout the epidermis, especially in the basal cell layer, and staining of hypopigmented area specimens displayed lower levels of melanin in the epidermis. Mutation analysis of the KITLG gene was performed, but no mutation was found.

STUDY LIMITATIONS: The new pathogenic gene was not found.

CONCLUSION: A family with FPHH was described. Analysis revealed that its members did not have any mutations of the KITLG gene, which provided evidence for genetic heterogeneity of this genodermatosis.
\end{abstract}

Keywords: Genetic heterogeneity; Hyperpigmentation; Hypopigmentation; Immunohistochemistry; Mutation

\section{INTRODUCTION}

Familial progressive hyperpigmentation (FPH) is an autosomal dominant disorder that was first reported in four affected individuals in two generations of an African-American Family. ${ }^{1}$ Its clinical signs consist of progressive, diffuse, partly blotchy hyperpigmented lesions presenting at birth, which increase in size and number with age. ${ }^{2}$ Cafe-au-lait macules and larger hypopigmented ash-leaf macules also appear in FPH. Familial progressive hyper- and hypopigmentation (FPHH) is distinct from FPH without hypopigmentation. Patients with FPHH often don't have systemic symptoms, but there is a familial pattern. ${ }^{3}$ A genome-wide linkage analysis of seven families with FPHH was performed, and it identified a linkage on 12 q21.12-q22 in a recent report. ${ }^{4}$ Then, the mutations of a single gene, KITLG, were discovered to cause various pigmentation disorders: FPH, FPHH, and likely dyschromatosis universalis hereditaria 2 (DUH2). The gain-of-function mutation of the KITLG gene affects melanin synthesis and causes familial progressive hyperpigmentation, and importantly, KITLG is expressed locally in the epidermal keratinocytes and endothelial cells of human skin. ${ }^{5,6}$ The KITLG gene and its receptor, KIT, play important roles in modulating melanocyte proliferation and pigment production. ${ }^{7}$ Here, we describe a Chinese

family with FPHH using clinical, histopathological, immunohistochemical (IHC) and mutation analyses.

\section{METHODS}

\section{Subjects}

A four-generation Chinese family had 14 individuals affected by FPHH ( 9 men and 5 women) and had pedigrees that showed an autosomal dominant inheritance pattern (Figure 1). The proband (III:7) was a 5-year-old girl. Extensive hyper- and hypopigmentation with irregular patches was found at one week of age, and over time, the patches progressed successively over the thigh, waist, back, chest, upper limb, and dorsum of the hands and feet, sparing only the face, palms, soles and oral mucosa. The hyperpigmentation was more intense at the age of 5 years than it was at 2 years, but there was no obvious change in the hypopigmentation (Figure 2). There were deep bronze-brown patches and irregular hypopigmented spots intermixed on her limbs and trunk without any systemic disease. The child was born to healthy, non-consanguineous parents following an uneventful pregnancy and eutocia. The family history

\section{Received on 11.01.2016.}

Approved by the Advisory Board and accepted for publication on 11.05.2016.

* Study conducted at the Department of Dermatology, Qilu Hospital of Shandong University - Jinan, Shandong Sheng, China.

Financial support: This study was surported by the Undergraduates Science and Technology Innovation Foundation of Shandong University (No. 2016428).

Conflict of interest: None

School of Medicine, Shandong University - Jinan, China.

Department of Dermatology, The First Hospital of HeBei Medical University - Shijiazhuang, China.

Department of Dermatology, Qilu Hospital of Shandong University - Jinan, Shandong Sheng, China. 
provided no evidence of chronic chemical poisoning or consanguineous marriage. For all patients, onset times were between one week and one month after birth, and the lesions deepened after exposure to the sun. None of the affected members of this family were found to have abnormalities in bone formation, neuro-psychomotor development, language, hearing or cognitive ability, skin cancer, or systemic disease.

Blood was drawn from ten affected and three unaffected individuals, and skin biopsies were taken from both the hyper- and hypopigmented lesions of the proband. Informed consent was obtained from all available patients and relatives for clinical and genetic investigation. The study was approved by the Institutional Ethical Committee of the hospital and was conducted according to the Declaration of Helsinki principles.

\section{Histopathological and IHC staining}

Skin biopsy specimens from the proband were stained with hematoxylin and eosin (HE). IHC staining for S100 and HMB45 was performed on formalin-fixed and paraffin-embedded sections of the lesions using the Elivision two-step system (Maixin Biotech Inc., Fuzhou, China). First, the tissue sections were deparaffinized, and they were rehydrated and subjected to microwave antigen retrieval in citrate buffer ( $\mathrm{pH}$ 6.0). Inhibition of endogenous peroxidase activity was accomplished by incubating the slices in $3 \% \mathrm{H}_{2} \mathrm{O}_{2}$ peroxidase inhibitor. After washing three times in phosphate buffered saline (PBS), the sections were incubated with a primary antibody against S100 or HMB45 (in operating fluid) at $4^{\circ} \mathrm{C}$ overnight. Then, the sections were incubated with a biotinylated secondary antibody at room temperature for $10 \mathrm{~min}$. Staining procedures were performed according to the manufacturer's instructions.

\section{Mutation analysis}

Genomic DNA was extracted from ten affected and three unaffected individuals using the Qiagen Blood DNA Kit (QIAGEN Inc., Valencia, CA, USA) according to the manufacturer's instructions. Screening for mutations in the KITLG gene was performed. We amplified the entire coding region of KITLG to span the open reading frame encoding for exons 1-9. After the reference sequence of the KITLG gene was obtained from the Genebank database, we produced the primer sequences using the Primer 3.0 program (Table 1). PCR was performed using $15 \mu$ of a reaction mixture that contained $20 \mathrm{ng}$ genomic DNA, $0.3 \mu \mathrm{M}$ each primer, $0.3 \mathrm{mM}$ dNTPs, 3.0 $\mathrm{mM} \mathrm{MgCl} 2$ and 0.1 units of Hotstar®Taq DNA polymerase (QIA-
GEN Inc., Valencia, CA, USA). Initial denaturation was conducted at $95^{\circ} \mathrm{C}$ for $15 \mathrm{~min}$, and denaturation for 40 cycles at $94^{\circ} \mathrm{C}$ for $40 \mathrm{~s}$ was then performed, followed by an annealing step at $58^{\circ} \mathrm{C}$ for $60 \mathrm{~s}$,
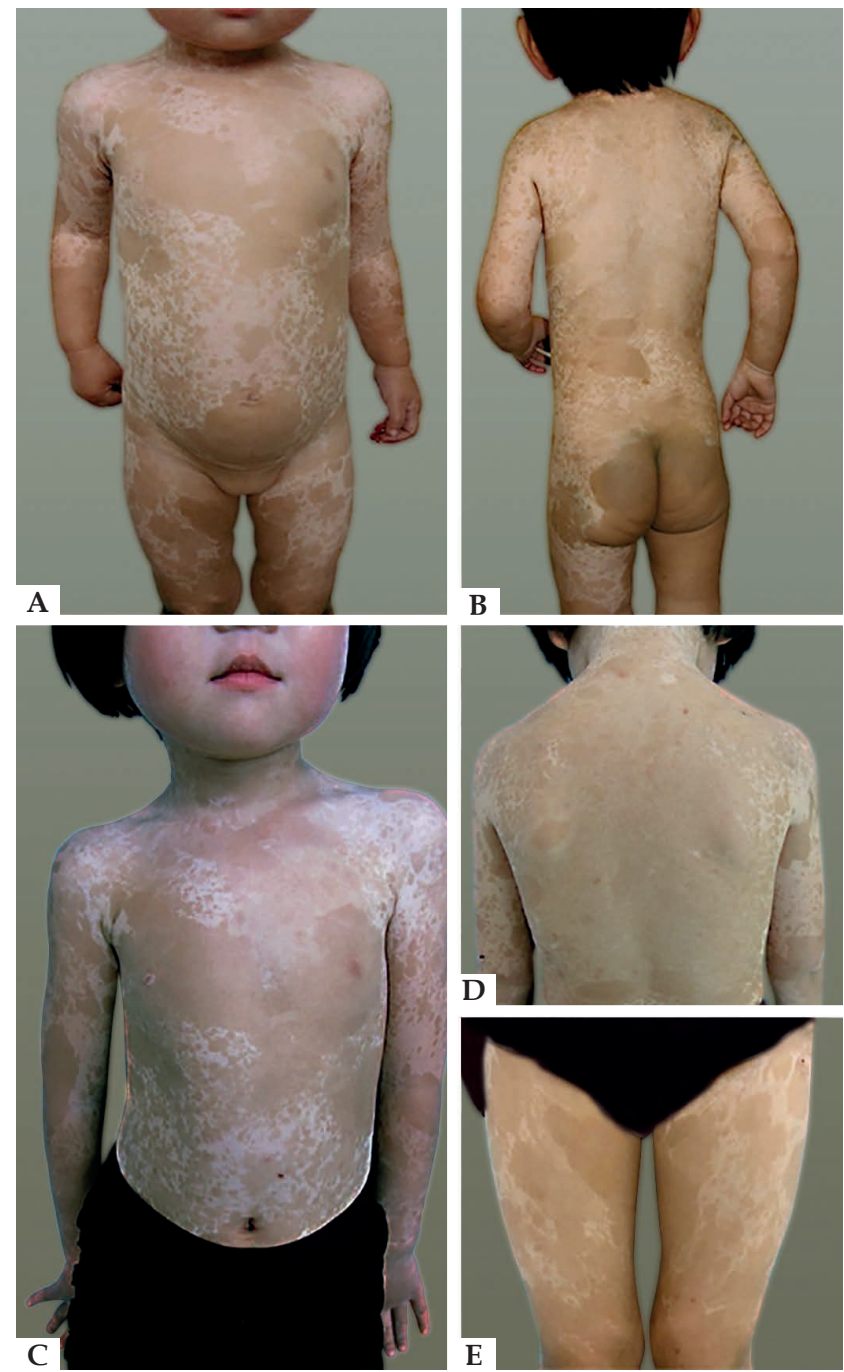

Figure 2: Clinical appearance of the proband. Clinical representations at 2 years old (A and B) showing hyper- and hypopigmented macules; Clinical representations at 5 years old (C, D and E) showing pigmented macules which were more serious

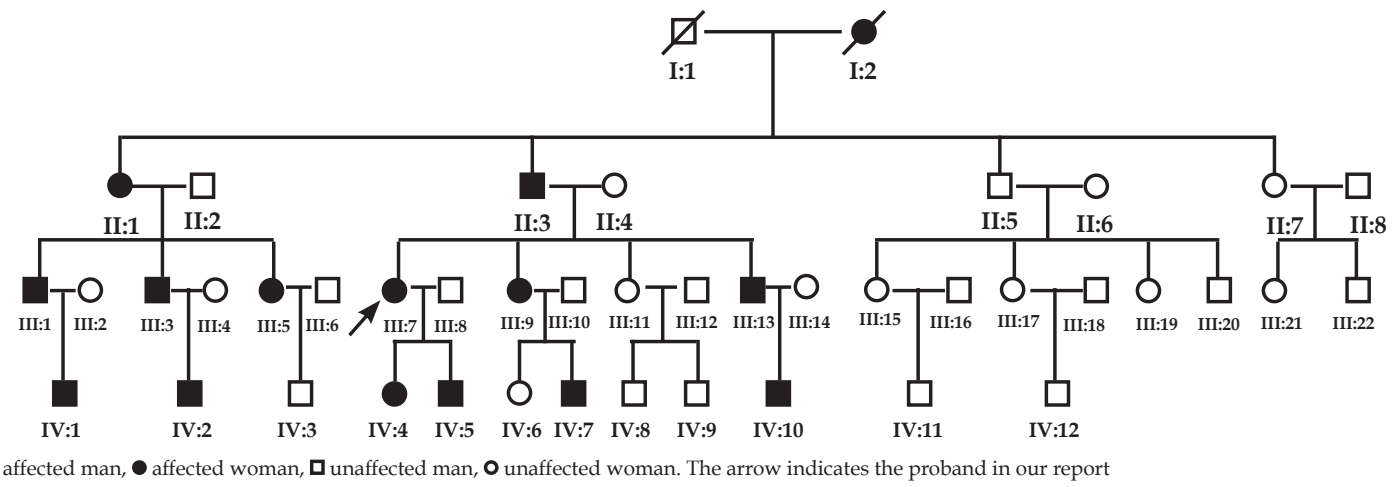

Figure 1: The pedigrees of the patients 
and an extension at $72^{\circ} \mathrm{C}$ for $55 \mathrm{~s}$, with a final extension at $72^{\circ} \mathrm{C}$ for $10 \mathrm{~min}$. After amplification, the products were purified using a QIAquick PCR Purification Kit (QIAGEN Inc., Valencia, CA, USA) and were directly sequenced on an Applied Biosystems 3730XL Sequencer (Carlsbad, CA, USA). Sequence comparisons and analysis were performed using the Chromas Version 12.0 software.

\section{RESULTS}

\section{Histopathological and IHC staining}

HE staining revealed a clear increase in melanin throughout the epidermis of the hyperpigmented tissue, particularly in the basal cell layer, and it revealed lower levels of melanin in the hypopigmented tissue. The S100 staining of hyperpigmented areas showed clear, dark staining in the nucleus and cytoplasm of both the epidermis and some dermis cells, especially in the basal layer.

\section{TABLE 1: Primers of the KITLG gene}

\begin{tabular}{llll} 
Exon & Forward primer & Reverse primer & $\begin{array}{l}\text { Fragment } \\
\text { length(bp) }\end{array}$ \\
\hline Exon1 & ggcctaatctgccaaacttct & aaaccgtctcctatgcaaagac & 543 \\
Exon2 & cttttgtgcatcaaggcatta & tttggggcaagaaaaatctaaa & 302 \\
Exon3 & tttgaaggatgcctttgatt & tatcctgatggtggagtgagtg & 556 \\
Exon4 & gcaggcacttgtaatctgagc & gcaggactgatttgcattgta & 579 \\
Exon5 & atcccagcttgttttaaggt & catttgaactggaaatgcttaca & 456 \\
Exon6 & cacttcacctttcagggactct & tttgtcagacacattccacaca & 476 \\
Exon7 & acaaccacagcataagagcaaa & ttcaagaagctgagtcacaatga & 581 \\
Exon8 & gcaaaaatcattgtgactcagc & ggatagagttgggactcactgg & 484 \\
Exon9 & tctgaccaagagcaaataagca & gccctaagcacattaactggac & 543 \\
& & &
\end{tabular}

These darkly stained regions indicated a large number of melanocytes and melanin and possibly Langerhans cells due to a lack of S100 specificity. HMB45 staining of the hyperpigmented tissue was similar to that of S100 in the same area with increased melanocytes and melanin, but there was little staining in the nucleus and dermis compared with S100. The staining for S100 and HMB45 were almost completely negative in the hypopigmentation areas. These findings were consistent with the HE staining (Figure 3). The diagnosis of FPHH was made based on the clinical features and family history.

\section{Mutation analysis}

The results from sequencing of ten affected and three unaffected individuals showed no causal mutations in the coding regions of the KITLG gene. We checked the experimental method and process several times to confirm this result.

\section{DISCUSSION}

$\mathrm{FPHH}$ is a rare congenital genodermatosis that is characterized by hyper- and hypopigmented patches in the skin. It presents in early infancy, and the patches increase in both size and number with age. ${ }^{5}$ Some studies on FPH and FPHH have been conducted around the world. The first family with FPH was reported to be an African-American family with four affected individuals across two generations. This report described the characteristics of hyperpigmentation and genodermatosis as well progressive skin pigmentation during the premenstrual period. ${ }^{1} \mathrm{~A}$ Chinese family that included 15 patients with FPH across six generations was described with the classical features, including extensive spots of the conjunctival tissue, face, neck, trunk, limbs, lips, oral mucosa, palms and soles. Increase in pigmentation was rapid during childhood and slowed
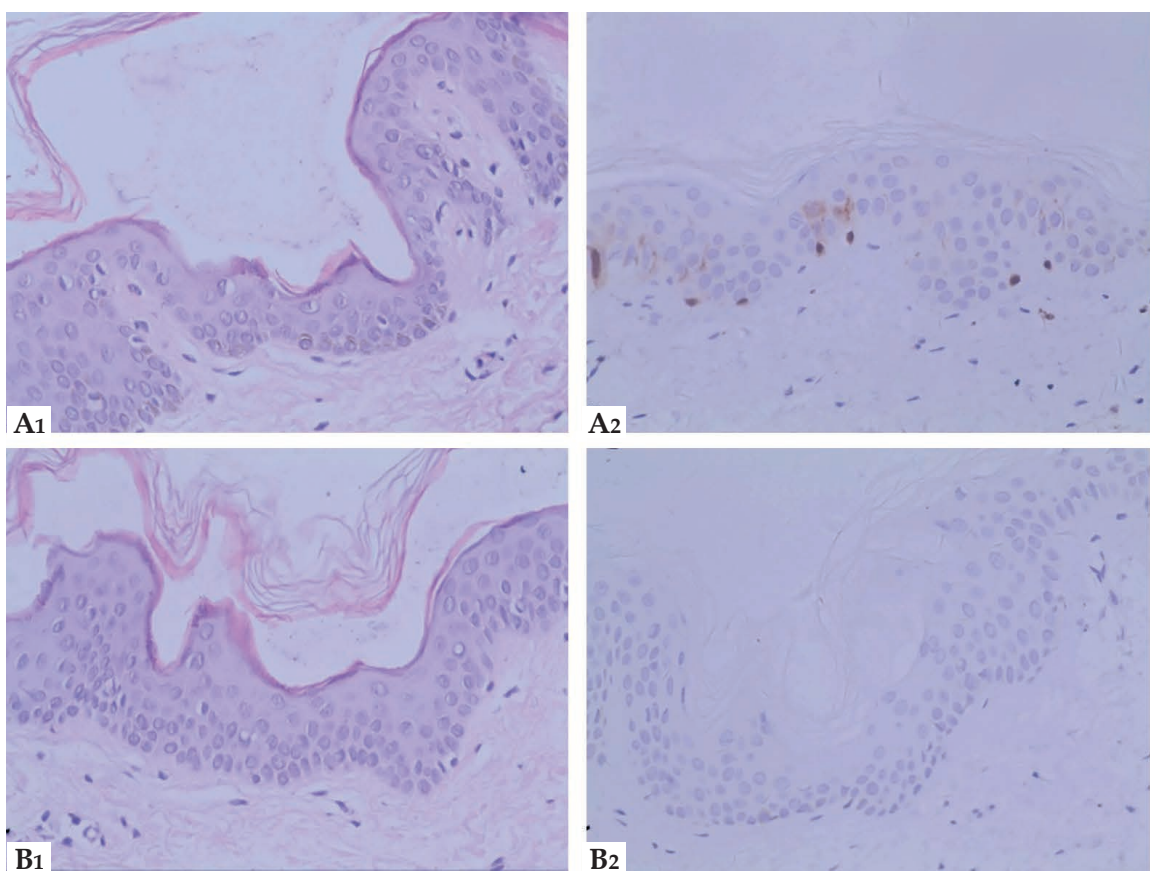
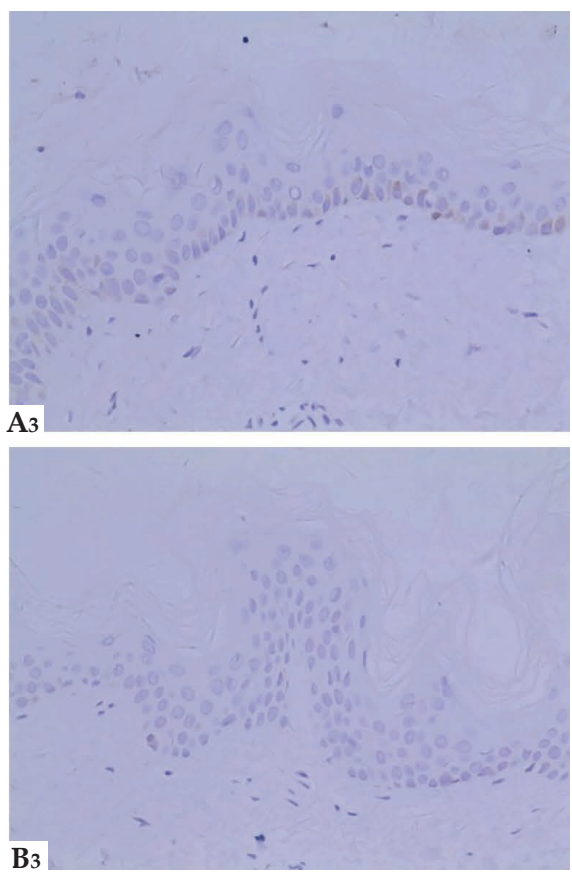

FIGURE 3: Histopathological and immunohistochemical features( Hematoxylin \& eosin x400). HE staining (A1), Immunohistochemical staining for S100 (A2) and HMB45 (A3) respectively from hyperpigmentation; HE staining (B1), Immunohistochemical staining for S100 (B2) and HMB45 (B3) respectively from hypopigmentation 
during adolescence. ${ }^{8}$ Five patients from three different families in southeast Germany presented with the symptoms of FPH, showing a specific pigment with dark complexions and incipient signs of blotchiness, but mainly diffuse hyperpigmentation intermixed with scattered small hypopigmented maculae and occasional cafe-au-lait spots. ${ }^{3}$ Indian monozygotic twins and a 17-year-old African-American male patient with FPH presented similarly to the other cases reported and showed no complications., ${ }^{9,10}$ A three-generation family with French-Canadian ancestry with individuals affected by FPHH was reported in 2005 to show concurrent tuberous sclerosis. ${ }^{11}$ Westerhof $e t$ al. described a three-generation family in which several family members were affected with hyper- and hypopigmented patches and had concurrent growth delay and mental deficiency.12 This complex of symptoms represented a new neurocutaneous syndrome distinct from tuberous sclerosis complex, which was then called Westerhof syndrome. Two yellow race siblings with Westerhof syndrome have also been reported. ${ }^{13}$ According to a recent report, a two-year-old Chinese girl with FPHH also had mild mental retardation, frequent epileptic seizures, impaired temperature regulation (hyperthermia) and increased susceptibility to infection. ${ }^{14}$ Two unrelated Caucasian families had six members affected with FPHH who had unusually sparse lateral eyebrows, two of whom had cancer. ${ }^{15}$

The patients from the family presented here gradually developed extensive hyper- and hypopigmentation over much of their bodies without any systemic disease, and the first presentation of the abnormal pigmentation occurred one week to one month after birth. The proband presented with mixed deep bronze-brown and irregular hypopigmented spots on her trunk and limbs. Hyperpigmented areas were contiguous and the hypopigmentation covered larger ranges with soya bean-sized hypopigmented spots. The skin of her face, palms, soles and oral mucosa were normal. In comparison with her symptoms at 2 years old, at 5 years old, we found that the hyperpigmented patches had further progressed in size and number, though only to a small degree. Menstruation did not appear to affect the disorder in women of this family. Due to the differences in race and complexion, the patients' phenotypes were not entirely the same as in the cases reported previously.

In this study, the histopathology of lesions presented that a marked increase in the amount of melanin in the basal cell layer of hyperpigmented area, and lower level of melanin in hypopigmented areas. The dermis was normal and no melanophages were seen. Keratinocytes had a larger number of melanin granules than normal and some of the granules displayed free melanosomes, but others within the phagosomes were displayed in a caucasoid pattern under electron microscopy. ${ }^{16}$ For ultrastructure, hypomelanotic skin showed small melanosomes within melanosome complexes in keratinocytes, on the contrary, hypermelanotic skin showed large melanosomes distributed singly in keratinocytes. ${ }^{12}$ In general, the histopathological features of this disease were similar in reports of different cases or families. . $^{1,3,5,8-10}$

IHC staining for S100 and HMB45 was also performed. S100 is a commonly used marker to stain Langerhans cells, melanocytes, and Pacinian corpuscles in dermatology. HMB45 recognizes mela- nocytes with a high degree of specificity. IHC staining for S100 and HMB45 showed clearly higher levels of melanin in hyperpigmented areas and the opposite result in hypopigmented areas, which was similar to the histopathology results in our study. Cardinali et al. performed IHC staining in tissue from two FPH patients and found an increased expression of hepatocyte growth factor (HGF), stem cell factor (SCF) and keratinocyte growth factor (KGF) in fibroblast-like cells of the upper dermis in hyperpigmented lesions. ${ }^{17}$ We did not found S100 and HMB45 staining in the previous studies of FPHH.

Based on the clinical and pathological presentation, the patients were diagnosed with FPHH. The patients in this family displayed massive hyper- and hypopigmentation lesions that spared only the face, palms, soles and oral mucosa and that showed different features from those presented in the majority of cases previously reported, but similar to those in a case reported by Zanardo. ${ }^{1,3,14}$ This suggests that different patterns of pigmentation exist in different cases. Differential diagnoses should be made regarding other diseases, especially cutis tricolor, such that FPHH is distinct from $\mathrm{FPH}$, in which no hypopigmented features are present. Dyschromatosis universalis hereditaria presents with an admixture of small pigmented flecks and spots with hypopigmented lesions in a generalized distribution. When localized to the extremities, this is called dyschromatosis symmetrica hereditaria or reticulate acropigmentation of Dohi. ${ }^{18}$ Incontinentia pigmenti shows hyperchromic and hypochromic lesions along the lines of Blaschko and follows a chronological progression through four distinct stages: vesicles on an erythematous base, verrucous hyperkeratotic lesions, hyperchromic spots, and hypochromic atrophic lesions. ${ }^{19}$

To find the gene that causes FPH, Wang et al ${ }^{5}$ performed a genome-wide scan for linkages in a previously described Chinese family with FPH. ${ }^{8}$ The locus was mapped to chromosome 12q21.31-q23.1 via two-point linkage analysis, and a gain-of-function missense mutation in the KITLG gene was found to be cosegregated in affected individuals in this family. In another report, linkage analysis was performed in seven families. Five of these families were related and were from southeastern Germany, whereas the other two were from America and Denmark. ${ }^{4}$ Three Germanic and one American family among these were described previously.,11 Multipoint analysis mapped the locus to chromosome 12q, and an analysis of two additional German families with FPHH showed the same result. ${ }^{3}$ Two heterozygous missense mutations were found in two unrelated Caucasian families with FPHH. ${ }^{15}$ This study confirmed that the gene underlying FPHH is the KITLG gene, which has also been demonstrated in separate studies. ${ }^{4,5}$

We performed a mutation analysis of the KITLG gene; however, this sequencing analysis did not show any mutation of the KITLG gene in the ten affected individuals. The experimental process was repeated several times, and the same results were found. Amyere et al. screened KITLG in seven families and did not find any mutation in the coding regions of KITLG in three of the families. ${ }^{4}$ Based on the typical clinical features and the fact that there were no mutations found in the coding regions of the KITLG gene in the family with FPHH presented here, we believe that FPHH is a heterogeneous genetic disease and that it is caused by more than one genetic locus. 


\section{CONCLUSION}

In conclusion, we described a multigenerational Chinese family with FPHH and investigated whether the KITLG gene played a causal role in the FPHH. We provided evidence of genetic heterogeneity in FPHH. To better understand the pathogenesis and genetic characteristics of KITLG, more studies, such as identifying potential new loci of FPHH using a genome-wide scan, are required. $\square$

\section{REFERENCES}

1. Chernosky ME, Anderson DE, Chang JP, Shaw MW, Romsdahl MM. Familial progressive hyperpigmentation. Arch Dermatol. 1971;103:581-91.

2. Zhang C, Deng Y, Chen X, Wu X, Jin W, Li H, et al. Linkage of a locus determining familial progressive hyperpigmentation (FPH) to chromosome 19p13.1-pter in a Chinese family. Eur J Dermatol. 2006;16:246-50.

3. Zanardo L, Stolz W, Schmitz G, Kaminski W, Vikkula M, Landthaler M, et al. Progressive hyperpigmentation and generalized lentiginosis without associated systemic symptoms: a rare hereditary pigmentation disorder in south-east Germany. Acta Derm Venereol. 2004;84:57-60.

4. Amyere M, Vogt T, Hoo J, Brandrup F, Bygum A, Boon L, et al. KITLG mutations cause familial progressive hyper- and hypopigmentation. J Invest Dermatol. 2011;131:1234-9.

5. Wang ZQ, Si L, Tang Q, Lin D, Fu Z, Zhang J, et al. Gain-of-function mutation of KIT ligand on melanin synthesis causes familial progressive hyperpigmentation. Am J Hum Genet. 2009;84:672-7.

6. Morita E, Lee DG, Sugiyama M, Yamamoto S. Expression of c-kit ligand in human keratinocytes. Arch Dermatol Res. 1994;286:273-7.

7. Hemesath TJ, Price ER, Takemoto C, Badalian T, Fisher DE. MAP kinase links the transcription factor Microphthalmia to c-Kit signalling in melanocytes. Nature. 1998;391:298-301.

8. Ling DB, Lo T. Familial progressive hyperpigmentation: a family study in $\mathrm{China}$. $\mathrm{Br}$ J Dermatol. 1991;125:607.

9. Gupta N, Sharma MC, Ramam M, Kabra M. Familial progressive hypermelanosis in Indian monozygotic twins. Pediatr Dermatol. 2011;28:62-5.

10. Yadav M, Ghonasgi S, Shah R, Meghana SM. Familial progressive hyperpigmentation: a case report. Case Rep Dent. 2012;2012:840167.

11. Hoo JJ, Shrimpton AE. Familial hyper- and hypopigmentation with age-related pattern change. Am J Med Genet A. 2005;132A:215-8.

12. Westerhof W, Beemer FA, Cormane RH, Delleman JW, Faber WR, de Jong JG, et al. Hereditary congenital hypopigmented and hyperpigmented macules. Arch Dermatol. 1978;114:931-6.

13. Vélez A, Salido R, Amorrich-Campos V, Garnacho-Saucedo G, Alvarez-López MA, Galán M, et al. Hereditary congenital hypopigmented and hyperpigmented macules (Westerhof syndrome) in two siblings. The Br J Dermatol. 2009;161:1399-400.

14. Zhang RZ, Zhu WY. Familial progressive hypo- and hyperpigmentation: a variant case. Indian J Dermatol Venereol Leprol. 2012;78:350-3.

\section{ACKNOWLEDGMENTS}

We gratefully acknowledge the patients' families for their participation in the study and for consenting the publication of clinical data and photos.
15. Cuell A, Bansal N, Cole T, Kaur MR, Lee J, Loffeld A, et al. Familial progressive hyper- and hypopigmentation and malignancy in two families with new mutations in KITLG. Clin Exp Dermatol. 2015;40:860-4.

16. Rebora A, Parodi A. Universal inherited melanodyschromatosis: a case of melanosis universalis hereditaria? Arch Dermatol. 1989;125:1442-3.

17. Cardinali G, Kovacs D, Giglio MD, Cota C, Aspite N, Amantea A, et al. A kindred with familial progressive hyperpigmentation-like disorder: implication of fibroblastderived growth factors in pigmentation. Eur J Dermatol. 2009;19:469-73.

18. Al Hawsawi K, Al Aboud K, Ramesh V, Al Aboud D. Dyschromatosis universalis hereditaria: report of a case and review of the literature. Pediatr Dermatol. 2002;19:523-6

19. Poziomczyk CS, Recuero JK, Bringhenti L, Maria FD, Campos CW, Travi GM, et al Incontinentia pigmenti. An Bras Dermatol. 2014;89:26-36.

\author{
MAILING ADDRESS: \\ Qing Sun \\ 107 Jinan Culture Road \\ Jinan, China. \\ E-mail: sunqing2015666@163.com \\ Yan-Jia Li \\ 89 Donggang Road, Shijiazhuang,China \\ Email: liyanjia2005@msn.com
}

How to cite this article: Fang X-K, He Y-X, Li Y-J, Chen L-R, Wang H-P, Sun Q. Familial progressive hyper- and hypopigmentation: a report on a Chinese family and evidence for genetic heterogeneity. An Bras Dermatol. 2017;92(3):329-33. 